TRANSACTIONS OF THE

AMERICAN MATHEMATICAL SOCIETY

Volume 196, 1974

\title{
GROUPS, SEMILATTICES AND INVERSE SEMIGROUPS. II
}

\author{
BY
}

\author{
D. B. McALISTER(1)
}

\begin{abstract}
An inverse semigroup is called proper if the equations ae = $e=e^{2}$ together imply $a^{2}=a$. In a previous paper, with the same title, the auxhor proved that every inverse semigroup is an idempotent separating homomorphic image of a proper inverse semigroup. In this paper a structure theorem is given for all proper inverse semigroups in terms of partially ordered sets and groups acting on them by order automorphisms. As a consequence of these two theorems, and Preston's construction for idempotent separating congruences on inverse semigroups, one can give a structure theorem for all inverse semigroups in terms of groups and partially ordered sets.
\end{abstract}

1. $P$-semigroups. If $\mathcal{X}$ is a partially ordered set and $A, B \in \mathcal{X}$ then the notation $A \wedge B \in \mathcal{X}$ means that $A$ and $B$ have a greatest lower bound in $\mathscr{X}$; this lower bound is denoted by $A \wedge B$. If $\mathcal{Y} \subseteq \mathcal{X}$ and $A, B \in \mathcal{Y}$ then the notation $A \wedge B \in \mathcal{Y}$. means that $A$ and $B$ have a greatest lower bound in $\mathcal{X}$ and that this lower bound $A \wedge B$ belongs to $\mathcal{Y}$. A nonempty subset $\mathcal{Y}$ of $\mathcal{X}$ is a subsemilattice of $\mathcal{X}$ if $A, B \in \mathcal{Y}$ imply $A \wedge B \in \mathcal{Y}$; $\mathcal{Y}$ is an ideal of $\mathcal{X}$ if $A \in \mathcal{Y}, B \in \mathcal{X}$ with $B \leq A$ implies $B \in \mathcal{Y}$.

Let $\mathcal{X}$ be a partially ordered set and let $\mathcal{Y}$. be a subsemilattice of $\mathcal{X}$. Suppose that $G$ is a group which acts on $\mathcal{X}$ by order automorphisms, on the left. Then it was shown in [6] that the set $P(G, \mathcal{X}, \mathcal{Y})$, of all pairs $(A, g) \in \mathcal{Y} \times G$ such that $A \wedge g B$ and $g^{-1}(A \wedge B)$ both exist and belong to $Y$ for all $B \in \mathcal{Y}$, is an inverse semigroup under the multiplication

$$
(A, g)(B, b)=(A \wedge g B, g h) .
$$

Indeed $S=P(G, \mathcal{X}, \mathcal{Y})$ is a proper inverse semigroup in the sense that the idempotents are a class modulo the congruence

$$
\sigma=\left\{(a, b) \in S \times S: e a=e b \text { for some } e^{2}=e \in S\right\} ;
$$

Munn [10] showed that $\sigma$ is the minimum group congruence on any inverse semigroup $S$.

Presented to the Society, January 16, 1974 under the title On the structure of inverse semigroups; received by the editors July 20, 1973.

AMS (MOS) subject classifications (197U). Primary $20 \mathrm{M10}$.

Key words and phrases. Inverse semigroup, proper inverse semigroup, semilattice, idempotent separating congruence, fundamental inverse semigroup, group action, order automorphisms.

(1) This research was supported by NSF Grant GP 27917.

Copyright $\odot 1974$, American Mathematical Society 
The following proposition contains several characterisations of proper inverse semigroups which we shall find useful in this paper.

Proposition 1.1. Let $S$ be an inverse semigroup with semilattice of idempotents $E$ and maximum group bomomorphic image $G=S / \sigma$. Then the following are equivalent:

(1) $S$ is proper;

(2) $\sigma \cap R=\Delta$ where $\Delta$ is the diagonal of $S \times S$;

(3) $\sigma^{4}$ is one-to-one on eacb $R$-class of $S$;

(4) the map $\phi: S \rightarrow E \times G$ defined by $s \phi=\left(s s^{-1}, s \sigma^{4}\right)$ is one-to-one;

(5) $a e=e=e^{2}$ implies $a^{2}=a$.

That $(1) \Rightarrow(2) \Rightarrow(4)$ was shown by Saitô [18]; the equivalence of (1) and (2) was shown by Reilly [15].

It follows from Proposition 1.1 that proper inverse semigroups are precisely those which can be coordinatised by their idempotents and maximum group homomorphic image.

Proper inverse semigroups are closed under products and inverse subsemigroups; this is immediate from Proposition 1.1(5). However, they are not closed under homomorphic images. Indeed the main theorem of [6] shows that every inverse semigroup is an idempotent separating homomorphic image of a full subsemigroup ([12, p. 159]) of a semigroup $P(G, X, Y)$ for some partially ordered set $X$ with subsemilattice $\mathcal{Y}$ and some group $G$ which acts on $\mathcal{X}$ by order automorphisms. Thus every inverse semigroup is an idempotent separating homomorphic image of a proper inverse semigroup.

Although the semigroups $P(G, \mathcal{X}, \mathcal{Y})$ and their full subsemigroups are manageable objects, the result that every inverse semigroup is an idempotent separating homomorphic image of a full subsemigroup of some $P(G, X, Y)$ has weaknesses. In the first place, although the semigroups $P(G, X, Y)$ themselves are explicitly described, their full subsemigroups are not. In the second, although the theorem gives an extensive class of proper inverse semigroups, it does not say how inclusive the class is.

Several examples of the semigroups $P(G, X, Y)$ are given in [6, [7], [8]; semigroups of this type had their genesis in Scheiblich's fundamental paper [17] on the structure of free inverse semigroups. The semigroups in these examples are rather special. In all of them, $\mathcal{Y}$. is an ideal of $\mathcal{X}$ as well as being a subsemilattice. Because of this,

$$
P=P(G, X, Y)=\left\{(A, g) \in \mathcal{Y} \times G: g^{-1} A \in \mathscr{Y}\right\},
$$

and the ideal structure of $P$ is considerably clearer than in the general case.

In the remainder of this paper, when we say that $P(G, X, Y)$ is a $P$-semigroup 
we will mean that $\mathcal{Y}$ is an ideal of $\mathcal{X}$ as well as being a subsemilattice.

Proposition 1.2 [G. Let $\mathcal{X}$ be a partially ordered set and let $\mathcal{Y}$ be an ideal and subsemilattice of $\mathcal{X}$. Let $G$ be a group wbich acts on $\mathcal{X}$ by order automoro phisms and suppose that $\mathcal{X}=G . \mathcal{Y}$. Then, in $P(G, X, Y)$,

(1) $(A, g)^{-1}=\left(g^{-1} A, g^{-1}\right)$;

(2) the idempotents are the elements $(A, 1), A \in Y_{\text {; they form a semilattice }}$ isomorpbic to $Y_{\text {; }}$

(3) $(A, g) \Re(B, b) \Leftrightarrow A=B ;(A, g) \mathcal{L}(B, b) \Leftrightarrow g^{-1} A=b^{-1} B$;

(4) $(A, g) \mathcal{H}(A, 1)$ if and only if $g A=A$; thus the $\mathcal{H}$-class of $(A, 1)$ is isomorpbic to the stabilizer of $A$ under $G$;

(5) $(A, 1) D(B, 1)$ if and only if $B=b A$ for some $b \in G$; thus there is a one-to-one correspondence between the set of orbits of $G$ and the set of D-classes of $P$;

(6) $(A, 1) \leq g(B, 1)$ if and only if there exists $g \in G$ sucb that $A \leq g B$;

(7) $(A, g) \sigma(B, b)$ if and only if $g=b$; thus $P / \sigma \approx G^{\prime}$ where $G^{\prime}=\lg \in G$ : $(A, g) \in P$ for some $A \in \mathcal{Y}\}=\{g \in G: g \mathcal{Y} \cap \mathcal{Y} \neq \square\}$.

Given $P$-semigroups $P(G, \mathcal{X}, \mathcal{Y})$ and $P(H, \mathcal{U}, \mathcal{O})$, with $\mathcal{Y}$ an ideal of $\mathcal{X}$ and $\mathcal{O}$ an ideal of $\mathcal{U}$, it is natural to ask under what conditions is $P(G, \mathcal{X}, \mathcal{Y}$ ) isomorphic to $P(H, \mathcal{U}, \mathcal{O})$. One way to ensure that this is the case is to require that the action of $G$ on $\mathcal{X}$ is equivalent to the action of $H$ on $\mathcal{U}$ in the sense that there is an order isomorphism $\theta$ of $\mathcal{X}$ onto $\mathcal{U}$, such that $\mathcal{Y} \theta=\mathcal{O}$, and an isomorphism $\phi$ of $G$ onto $H$ such that the diagram

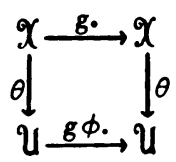

commutes for each $g \in G$. If the actions are equivalent, then the mapping $\psi$ : $P(G, X, Y) \rightarrow P(H, \mathcal{U}, \mathcal{Y})$ defined by $(A, g) \psi=(A \theta, g \phi)$ is an isomorphism of $P(G, \mathcal{X}, \mathcal{Y})$ onto $P(H, \mathcal{U}, \mathcal{O})$.

An element $g \in G$ appears in an element of $P(G, X, Y)$ if and only if $g^{-1} B \in \mathcal{Y}$ for some $B \in \mathcal{Y}$; that is, $g^{\mathcal{Y}} \cap \mathcal{Y} \neq \square$. Similarly, $A \in \mathcal{X}$ appears in an element of $P(G, X, Y)$ only in the form $g B$ for some $g \in G$ such that $(B, g) \in P$ for some $B \in Y$. If we let

$$
G^{\prime}=\left\{g \in G: \text { Y } \cap g^{\mathcal{Y}} \neq \square\right\}, \quad x^{\prime}=G . Y .,
$$

then $G^{\prime}$ is a group and $X^{\prime}$ is a partially ordered set having $Y$ as an ideal and subsemilattice. Further $P(G, X, Y)=P\left(G^{\prime}, X^{\prime}, Y_{1}\right)$. Hence when dealing with $P$. semigroups, no loss of generality is entailed by requiring

$$
\text { (P.1) G.Y. }=\mathfrak{X} \text {, }
$$


(P.2) for each $g \in G, g y \cap \mathcal{Y} \neq \square$.

With the restrictions that (P.1) and (P.2) should hold, it turns out that $P(G, \mathcal{X}, \mathcal{Y}) \approx P(H, \mathcal{U}, \mathcal{O})$ if and only if the actions of $G$ on $\mathcal{X}$ and $H$ on $\mathcal{U}$ are equivalent.

Theorem 1.3. ' Let $X_{i}$ be a partially ordered set witb $\mathcal{Y}_{i}$ an ideal and sub. semilattice of $X_{i}$ and let $G_{i}$ be a group whicb acts on $X_{i}$ by order automorphisms sucb that $G_{i} Y_{i}=X_{i}$ and, for each $g \in G_{i}, g Y_{i} \cap Y_{i} \neq \square, i=1,2$. Then $P\left(G_{1}, X_{1}, Y_{1}\right) \approx P\left(G_{2}, X_{2}, Y_{2}\right)$ if and only if the action of $G_{1}$ on $X_{1}$ is equivalent to the action of $G_{2}$ on $\mathcal{X}_{2}$.

Proof. We have already seen that, if the actions are equivalent the semigroups are isomorphic so suppose that $\psi$ is an isomorphism of $P\left(G_{1}, X_{1}, Y_{1}\right)$ onto $P\left(G_{2}, X_{2}, Y_{2}\right)$.

Because (P.1) and (P.2) hold, for $P\left(G_{1}, X_{1}, \mathscr{Y}_{1}\right)$ and $P\left(G_{2}, X_{2}, Y_{2}\right)$, it follows from Proposition 1.2(7) that there is an isomorphism $\phi: G_{1} \rightarrow G_{2}$ such that the diagram

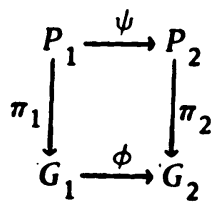

commutes where $\pi_{i}$ is the projection $P_{i} \rightarrow G_{i}, i=1,2$. Further, since $\psi$ is an isomorphism of $P_{1}$ onto $P_{2}$, the map $\theta$ defined by $(A, 1) \psi=(A \theta, 1)$ is an isomorphism of $\mathcal{Y}_{1}$ onto $\mathcal{Y}_{2}$.

Let $(A, g) \in P$; then, since $\pi_{1} \phi=\psi \pi_{2},(A, g) \psi=(B, g \phi)$ for some $B \in Y_{1}$. But $(A, g) R(A, 1)$ implies $(B, g \phi)=(A, g) \psi R(A, 1) \psi=(A \theta, 1)$ so $B=A \theta$. Thus $(A, g) \psi=(A \theta, g \psi)$ for all $(A, g) \in P$.

Suppose $x A \leq y B$ in $X_{1}$, where $A, B \in \mathcal{Y}_{1}$ then $y^{-1} x A \leq B$ so that $\left(A, x^{-1} y\right) \in P_{1}$. Now $\left(A, x^{-1} y\right)^{-1}\left(A, x^{-1} y\right)=\left(y^{-1} x A, 1\right) \leq(B, 1)$ so that

$$
(B \theta, 1)=(B, 1) \psi \geq\left[\left(A, x^{-1} y\right) \psi\right]^{-1}\left(A, x^{-1} y\right) \psi=\left(\left(y^{-1} x\right) \phi A \theta, 1\right)
$$

since $\psi$ is an isomorphism. Hence $B \theta \geq\left(y^{-1} x\right) \phi A \theta$; that is $x \phi A \theta \leq y \phi B \theta$. Dually, $x \phi A \theta \leq y \phi B \theta$ implies $x A \leq y B$. Hence, since (P.1) holds for $P_{1}$ and $P_{2}$, we can extend $\theta$ to an order isomorphism of $X_{1}$ onto $X_{2}$ by defining $(x A) \theta=$ $x \phi A \theta$ for each $x \in G_{1}, A \in Y_{1}$. Further, from the definition of $\theta$,

$$
\{g .(x A)\} \theta=\{(g x) A\} \theta=(g x) \phi A \theta=g \psi(x A) \theta
$$

for each $A \in Y_{1}, g, x \in G_{1}$. Hence the action of $G_{1}$ on $\mathscr{X}_{1}$ is equivalent to that of $G_{2}$ on $X_{2}$. Indeed $(A, g) \psi=(A \theta, g \phi)$ for $(A, g) \in P_{1}$. 
2. Proper inverse semigroups, Throughout this section, $S$ will denote a proper inverse semigroup with semilattice of idempotents $E$ and maximum group homomorphic image $G=S / \sigma$. We shall prove that there is a partially ordered set $\mathcal{X}$ having an ideal and subsemigroup $\mathcal{Y}$ and a group $G^{\prime}$ which acts on $\mathcal{X}$ by order automorphisms such that $S \approx P\left(G^{\prime}, X, Y\right)$; in fact, conditions (P.1) and (P.2) will also hold. By Proposition 1.2, $Y$ must be isomorphic to $E$ and $G^{\prime}$ must be isomorphic to $G$ so that the real problem is to find $X$. Indeed, by Theorem 1.3, the solution we obtain is unique up to equivalent actions.

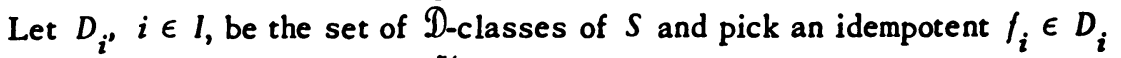
for each $i \in I$; denote by $H_{i}$ the $\mathcal{H}$-class containing $f_{i}$. Further, for each $i \in I$,

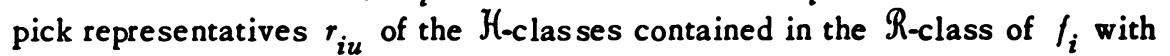
$f_{i}$ the representative of its class; denote this set of representatives by $E_{i}$. Then

(1) each element of $S$ can be uniquely expressed in the form $r_{i u}^{-1} b_{i} r_{i v}$ where $r_{i u}, r_{i v} \in E_{i}, b_{i} \in H_{i}$ for some $i \in I$;

(2) the idempotents of $S$ are precisely the elements $r_{i u}^{-1} r_{i u}=f_{i u}, r_{i u} \in E_{i}$, $i \in I$; they are all distinct.

This type of coordinatisation of $S$ underlies most structure theorems for inverse semigroups. Ours is no exception.

For each $i \in I$ we shall denote by $G_{i}$ the subgroup $H_{i} \sigma^{4}$ of $G$; similarly, we shall denote by $g_{i}$ the image in $G$ of $b_{i} \in H_{i}$. Since $S$ is proper, $\sigma^{4}$ is oneto-one on $H_{i}$ so that $G_{i} \approx H_{i}$. Further, let $k_{i u}$ denote the image in $G$ of $r_{i u} \epsilon$ $E_{i}, i \in I$. Since $S$ is proper, Proposition 1.1, shows that $\sigma^{4}$ is one-to-one on the

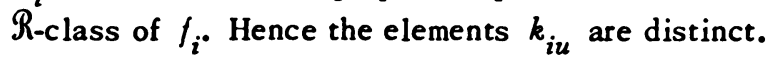

Finally, for each $i, j \in I$, let $B_{i j}=\left\{k_{j u} \in G: r_{j u}^{-1} r_{j u} \leq f_{i}\right\}$. Since $S$ is proper, for each $k \in B_{i j}$, there is a unique element in the R-class of $f_{j}$ which is mapped onto $k$. Hence, given $B_{i j}$, we can recover those idempotents $e \in D_{j}$ with $e \leq f_{i^{*}}$ The set $B_{i j}$ may be empty. For example, if $S$ is a semilattice of groups, $B_{i j} \neq \square$ if and only if $f_{i} \geq f_{j}$

Lemma 2.1. $f_{i u} \geq f_{j v} \Leftrightarrow k_{j v} \equiv k_{j \omega} k_{i u}\left(G_{j}\right)$ for some $k_{j \omega} \in B_{i j}$

Proof. $f_{i u} \geq f_{j v}$ implies $r_{j v}=r_{j v} f_{i u}$. Now

$$
r_{j v} r_{i u}^{-1}\left(r_{j v} r_{i u}^{-1}\right)^{-1}=r_{j v} r_{i u}^{-1} r_{i u} r_{j v}^{-1}=r_{j v} r_{j v}^{-1}=f_{j}
$$

since $f_{i u} \geq f_{j v}$; thus $r_{j v} r_{i u}^{-1} R f_{j}$. Hence $r_{j v} r_{i u}^{-1}=b_{j} r_{j \omega}$ for some $b_{j} \in H_{j}$ and and $r_{j \omega} \in E_{j}$. Further

$$
r_{j \omega}^{-1} r_{j \omega}=r_{j \omega}^{-1} b_{j}^{-1} b_{j} r_{j \omega}=r_{i u} r_{j v}^{-1} r_{j v} r_{i u}^{-1} \leq r_{i u} r_{i u}^{-1}=f_{i}
$$

Thus, in $G$ we have $k_{j v}=g_{j} k_{j \omega} k_{i u} \equiv k_{j \omega} k_{i u}\left(G_{j}\right)$ where $k_{j \omega} \in B_{i j}$ 
Conversely, suppose that $k_{j v}=g_{j} k_{j \omega} k_{i u}$ where $g_{j} \in G_{j}, k_{j \omega} \in B_{i j}$ Consider $b_{j} \boldsymbol{r}_{j} \boldsymbol{r}_{i u}$ in $S$. Then

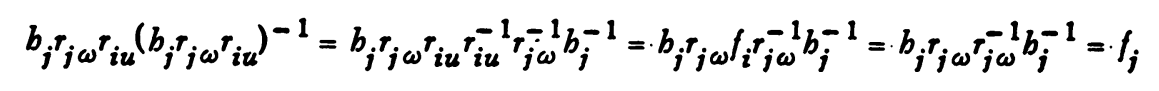

since $f_{j \omega} \leq_{i i^{*}}$. Thus $h_{j} r_{j \omega} \boldsymbol{r}_{i u} R r_{j v^{*}}$. Since, $S$ is proper and $r_{j v}$ and $b_{j} r_{j \omega}{ }^{r_{i u}}$ have the same image in $G$, it follows, from Proposition 1.1(2), that $r_{j v}=b_{j} r_{j} \omega^{r_{i u}}{ }^{*}$ Hence $f_{j v} \leq f_{i u^{*}}$

Lemma 2.1 shows that it is possible to reconstruct the partial order in $E$ in terms of $G$. Indeed, if we let $y$ be the set of all pairs $\left(i, G_{i} k_{i u}\right), i \in I, k_{i u} \in$ $E_{i} \sigma^{4}$, with the partial ordering

$$
\left(i, G_{i} x\right) \geq\left(j, G_{j} y\right) \Leftrightarrow y \equiv k_{j \omega^{x}}\left(G_{j}\right)
$$

for some $k_{j \sigma} \in B_{i j}$, then Lemma 2.1 shows that $E \approx Y$.

This identification of $\mathcal{Y}$ leads to a natural candidate for $X$ in $P(G, X, Y)$; namely the set $\bigcup\left\{\{i\} \times G / G_{i}: i \in I\right\}$ with

$$
\left(i, G_{i} x\right) \geq\left(j, G_{j} y\right) \Leftrightarrow y \equiv k_{j \omega} x\left(G_{j}\right)
$$

for some $k_{j \omega} \in B_{i j}$. It also leads to a natural action of $G$ on $X_{;}$namely g. $\left(i, G_{i} x\right)=\left(i, G_{i} \times g^{-1}\right)$. We shall show that $X$ is a partially ordered set having $\mathcal{Y}$ as an ideal and subsemilattice. To prove this, we need two technical lemmas.

Lemma 2.2. If $k_{i u} g_{j} k_{j v} \in G_{i}$ for some $k_{i u} \in B_{j i}, k_{j v} \in B_{i j}$ and $g_{j} \in G_{j}$ then $i=j$ and $k_{j v}=1$.

Proof. Suppose $k_{i u} g_{j} k_{j v} \in G_{i}$ and consider $r_{i u} b_{j} r_{j v}$. Then

$$
r_{i u} b_{j} r_{j v} r_{j v}^{-1} b_{j}^{-1} r_{i u}^{-1}=r_{i u} f_{j} r_{i u}^{-1}=r_{i u} r_{i u}^{-1}=\cdot f_{i}
$$

since $k_{i u} \in B_{j i}$. Thus, if $k_{i u} g_{j} k_{j v}=g_{i}$ we have $\left(b_{i}, r_{i u} b_{j} r_{j v}\right) \in \sigma \cap R$ whence, by Proposition 1.1, $b_{i}=r_{i u} b_{j} r_{j v^{*}}$. Since $k_{j v} \in B_{i j}$, this gives $f_{i}=f_{i} r_{j v}^{-1} r_{j v} \leq$ $r_{j v}^{-1} r_{j v} \leq f_{i}$ so that $f_{i}=r_{j v}^{-1} r_{j v} D f_{j}$. Hence $i=j$ and $f_{i}=f_{j}$. But then $r_{j v}^{-1} r_{j v}=$ $f_{i}=f_{j}=r_{j v} r_{j v}^{-1}$ so that $r_{j v} \mathcal{H} f_{j}$. Since $f_{j}$ is the representative of its $\mathcal{H}_{\text {-class, }}$ this implies $r_{j v}=f_{j}$. Thus $k_{j v}=1$.

Lemma 2.3. If $k_{j v} \in B_{i j}, k_{n \omega} \in B_{j n}$ and $g_{j} \in G_{j}$ then $k_{n \omega} g_{j} k_{j v} \equiv k_{n u}\left(G_{n}\right)$ for some $k_{n u} \in B_{i n^{*}}$

Proof. In $S$,

$$
r_{n \omega} b_{j} r_{j \nu} r_{j \nu}^{-1} b_{j}^{-1} r_{n \omega}^{-1}=r_{n \omega} f_{j}^{r_{n \omega}^{-1}}=r_{n \omega} r_{n \omega}^{-1}=f_{n}
$$

since $k_{n \omega} \in B_{j n}$ implies $r_{n \omega}^{-1}{ }_{n \omega} \leq f_{j}$. 
Now

Thus $r_{n \omega} b_{j} r_{j v} \Re f_{n}$ so that $r_{n \omega} b_{j} r_{j v}=b_{n} r_{n u}$ for some $b_{n} \in H_{n^{\circ}} r_{n u} \in E_{n}$.

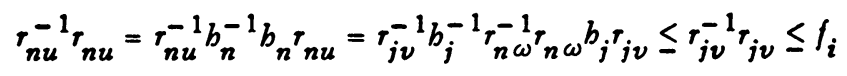

since $k_{j v} \in B_{i j}$. Hence $k_{n u} \in B_{i n}$ and so $k_{n \omega} g_{j} k_{j v}=g_{n} k_{n u} \equiv k_{n u}\left(G_{n}\right)$ where $k_{n u} \in B_{i n}$.

It follows from this lemma that the relation

$$
\left(j, G_{j} x\right) \geq\left(n, G_{n} y\right) \Leftrightarrow y \equiv k_{n \omega} x\left(G_{n}\right)
$$

for some $k_{n \omega} \in B_{j n}$, is well defined on $X$. For suppose $G_{j} x=G_{j} z, G_{n} y=G_{n} v$ so that $x=g_{j} z, v=g_{n} y$ for some $g_{j} \in G_{j}, g_{n} \in G_{n}$. Then $\left(j, G_{j} x\right) \geq\left(n, G_{n} y\right)$ implies $y \equiv k_{n \omega} x\left(G_{n}\right)$ for some $k_{n \omega} \in B_{j n}$; thus $v \equiv k_{n \omega} g_{j} z\left(G_{n}\right)$. But, since $f_{j}$ is the representative of its $\mathcal{H}_{\text {-class, }} 1 \in B_{j j}$ so that Lemma 2.3 shows $k_{n \omega} g_{j}=$. $k_{n \omega} g_{j} 1 \equiv k_{n u}\left(G_{n}\right)$ for some $k_{n u} \in B_{j n}$. Hence $v \equiv k_{n u} z\left(G_{n}\right)$ where $k_{n u} \in B_{j n}$; that is $\left.\left(j, G_{j} z\right) \geq n, G_{n} v\right)$. Similarly, $\left(j, G_{j} z\right) \geq\left(n, G_{n} v\right)$ implies $\left(j, G_{j} x\right) \geq\left(n, G_{n} y\right)$ so the definition of $\geq$ is independent of the particular coset representatives; indeed it depends only on the choice of idempotents $f_{i}, i \in I$.

Lemma 2.4. $X$ is a partially ordered set baving 9 as an ideal and subsemilattice.

Proof. First of all, since $f_{i} \geq f_{i}=f_{i}^{-1} f_{i}, 1 \in B_{i i}$ for each $i \in I$. Hence $\geq$ on $\mathcal{X}$ is reflexive.

Next, suppose that $\left(i, G_{i} x\right) \geq\left(j, G_{j} y\right) \geq\left(i, G_{i} x\right)$. Then $y=g_{j} k_{j v} x, x=g_{i} k_{i u} y$ for some $g_{i} \in G_{i}, g_{j} \in G_{j}, k_{j v} \in B_{i j}, k_{i u} \in B_{j i}$. Thus $x=g_{i} k_{i u} g_{j} k_{j v} x$ so that $k_{i u} g_{j} k_{j v} \in G_{i}$. Hence, by Lemma $2.2, i=j$ and $k_{j v}=1$ which implies $\left(i, G_{i} x\right)=$ $\left(j, G_{j} y\right)$. Thus $\geq$ is antisymmetric.

Suppose now that $\left(i, G_{i} x\right) \geq\left(j, G_{j} y\right) \geq\left(n, G_{n} z\right)$. Then $y=g_{j} k_{j v} x$ and $z=$ $g_{n} k_{n \omega} y$ for some $g_{j} \in G_{j}, g_{n} \in G_{n}, k_{j v} \in B_{i j}$ and $k_{n \omega} \in B_{j n}$. Thus $z=$ $g_{n} k_{n \omega} g_{j} k_{j v} x_{0}$ By Lemma 2.3, $k_{n \omega} g_{j} k_{j v} \equiv k_{n u}\left(G_{n}\right)$ for some $k_{n u} \in B_{i n}$ so $z \equiv$ $k_{n u} x\left(G_{n}\right)$. Hence $\left(i, G_{i} x\right) \geq\left(n, G_{n} z\right)$ so that $\geq$ is transitive.

Since $Y$ is known to be a semilattice, by Lemma 2.1, the proof will be complete when we show that $\mathcal{Y}$ is an ideal of $\mathcal{X}$.

Suppose that $\left(i, G_{i} k_{i u}\right) \geq\left(j, G_{j} y\right)$; then $y \equiv k_{j v} k_{i u}\left(G_{j}\right)$ for some $k_{j v} \in B_{i j}$ But, since $k_{j v} \in B_{i j}$

$$
r_{j v}{ }^{r}{ }_{i u}^{r_{i u}^{-1}} r_{j v}^{-1}=r_{j v} f_{i} r_{j v}^{-1}=r_{j v}{ }_{j v}^{-1}=f_{j},
$$

so that $r_{j v} \boldsymbol{r}_{i u}=b_{j} \boldsymbol{r}_{j \omega}$ for some $b_{j} \in H_{j}, r_{j \omega} \in E_{j}$. Applying $\sigma^{\natural}$, we obtain $y \equiv$ $k_{j \omega}\left(G_{j}\right)$ so that $\left(j, G_{j} y\right)=\left(j, G_{j} k_{j \omega}\right) \in \mathcal{Y}$. Hence $Y$ is an ideal of $X$.

From the form of the partial order on $\mathcal{X}$, the natural action of $G$ by $g \cdot\left(i, G_{i} x\right)=$ 
$\left(i, G_{i} x g^{-1}\right)$ is clearly by order automorphisms. Hence we can construct $P(G, X, Y)$. For any $\left(i, G_{i} k_{i u}\right) \in \mathcal{Y}$,

$$
\begin{aligned}
\left(\left(i, G_{i} k_{i u}\right), g\right) \in P & \Leftrightarrow g^{-1}\left(i, G_{i} k_{i u}\right) \in Y \\
& \Leftrightarrow G_{i} k_{i u} g=G_{i} k_{i v} \text { for some } k_{i v} \in E_{i} \sigma^{4} \\
& \Leftrightarrow g \in k_{i u}^{-1} G_{i} k_{i \nu} \subseteq D_{i} \sigma^{4} .
\end{aligned}
$$

It follows that one can define a map $\psi: S \rightarrow P(G, X, Y)$ by

$$
\left(r_{i u}^{-1} b_{i} r_{i v}\right)=\left(\left(i, G_{i} k_{i u}\right), k_{i u}^{-1} g_{i} k_{i v}\right)
$$

and that $\psi$ is onto.

Let $s=r_{i u}^{-1} b_{i} r_{i v}$ and $t=r_{j x}^{-1} b_{j} r_{j v}$ be elements of $S$. Then $s s^{-1}=r_{i u}^{-1} r_{i u}$, $t t^{-1}=r_{j x}^{-1} r_{j x}$ so that, by Lemma $2.1, s \psi=t \psi$ if and only if $s s^{-1}=t t^{-1}$ and $s \sigma^{\natural}=t \sigma^{\natural}$. Since $S$ is proper, this implies $s=t$ so that $\psi$ is also one-to-one.

Lemma 2.5. $\psi$ is a bomomorphism.

Proof. Let $s=r_{i u}^{-1} b_{i} r_{i v}$ and $t=r_{j x}^{-1} b_{j} r_{j y}$ be elements of $S$. Then

$$
s t(s t)^{-1}=r_{i u}^{-1} b_{i} r_{i v} r_{j x}^{-1} r_{j x} r_{i v}^{-1} b_{i}^{-1} r_{i u}=r_{i u}^{-1} b_{i} r_{i v} r_{n \omega}^{-1} r_{n} \omega_{i v}^{r^{-1} b_{i}^{-1} r_{i u}}
$$

where $r_{n \omega}^{-1} r_{n \omega}=r_{i v}^{-1} r_{i v} r_{j x}^{-1} r_{j x}$.

Now

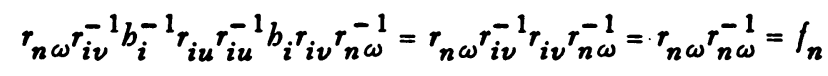

since $r_{n \omega}^{-1} r_{n \omega} \leq r_{i v}^{-1} r_{i v}$. Thus $r_{n \omega} r_{i \nu}^{-1} b_{i}^{-1} r_{i u}=b_{n} r_{n z}$ for some $b_{n} \in H_{n}$ and $r_{n z} \epsilon$ $E_{n}$ so that $s t=r_{n z}^{-1} k_{n} r_{n c}$ for some $k_{n} \in H_{n}, r_{n c} \in E_{n}$.

Hence

$$
\begin{aligned}
s t \psi & =\left(\left(n, G_{n} k_{n z}\right), s t \sigma^{4}\right)=\left(\left(n, G_{n} k_{n \omega} k_{i v}^{-1} g_{i}^{-1} k_{i u}\right), s t \sigma^{4}\right) \\
& =\left(k_{i u}^{-1} g_{i} k_{i v}\left(n, G_{n} k_{n \omega}\right), s t \sigma^{4}\right)=\left(s \sigma^{4}\left\{\left(i, G_{i} k_{i v}\right) \wedge\left(j, G_{j} k_{j x}\right)\right\}, s t \sigma^{4}\right)
\end{aligned}
$$

since, by Lemma $2.1, y \approx E$,

$$
\begin{aligned}
& =\left(\left(i, G_{i} k_{i u}\right) \wedge s \sigma^{\natural}\left(j, G_{j} k_{j x}\right), s t \sigma^{\natural}\right) \\
& =\left(\left(i, G_{i} k_{i u}\right), s \sigma^{\natural}\right)\left(\left(j, G_{j} k_{j x}\right), t \sigma^{\natural}\right)=s \psi t \psi .
\end{aligned}
$$

We have thus proved the converse part of the following theorem; the direct part is immediate from Proposition 1.2.

Theorem 2.6. Let $\mathcal{X}$ be a partially ordered set and let $\mathcal{Y}$ be an ideal and subsemilattice of $\mathcal{X}$. If $G$ is a group which acts on $\mathcal{X}$ by order is omorphisms 
then $P(G, X, Y)$ is a proper inverse semigroup.

Conversely, any proper inverse semigroup is isomorphic to $P(G, X, Y)$ for some partially ordered set $\mathcal{X}$ with ideal and subsemilattice $\mathcal{Y}$ and some group $G$ which acts on $\mathcal{X}$ by order automorphisms.

The partially ordered set $\mathcal{X}$ in the proof of Theorem 2.6 was manufactured from the group $G$. Further, since $X=G . Y$ and $g . Y \cap Y \neq \square$ for each $g \in G$, The orem 1.3 shows that $\mathcal{X}$ is unique up to equivalence of actions. The proof of Theorem 2.6 was designed so that we could abstractly characterise $X$ in terms of $G$ and could thus obtain all proper inverse semigroups in terms of groups.

Theorem 2.7. Let $G$ be a group and let $\left\{G_{i}: i \in I\right\}$ be a set of subgroups of $G$. For each $i \in I$, let $E_{i}$ be a set of elements $k_{i u}$ of $G$ such that the cosets $G_{i} k_{i u}$ are distinct. Further, for each $i, j \in I$, let $B_{i j}$ be a subset of $E_{j}$ sucb that the following bold

(1) $1 \in B_{i i}$ for each $i \in I$;

(2) if $k_{i u} g_{j} k_{j v} \in G_{i}$ for $s$ ome $k_{i u} \in B_{j i}, k_{j v} \in B_{i j}$ and $g_{j} \in G_{j}$ then $i=j$ and $k_{j v}=1$;

(3) if $k_{j v} \in B_{i j}, k_{n \omega} \in B_{j n}$ and $g_{j} \in G_{j}$ then $k_{n \omega} g_{j} k_{j v} \equiv k_{n u}\left(G_{n}\right)$ for some $k_{n u} \in B_{i n}$

(4) if $k_{j v} \in B_{i j}, k_{i u} \in E_{i}$ then $k_{j v} k_{i u} \equiv k_{j \omega}\left(G_{j}\right)$ for some $k_{j \omega} \in E_{j}$

Then the set $\mathcal{X}=\bigcup\left\{\{i\} \times G / G_{i}: i \in I\right\}$ is partially ordered by

$$
\left(i, G_{i} x\right) \geq\left(j, G_{j} y\right) \Leftrightarrow y \equiv k_{j v} x\left(G_{j}\right) \text { for some } k_{j v} \in B_{i j}
$$

Further the set $\mathcal{Y}=\left\{\left(i, G_{i} k_{i u}\right): k_{i u} \in E_{i}, i \in I\right\}$ is an ideal of $X_{\text {. Suppose that }}$

(5) $Y$ is a semilattice under $\leq$ and let $G$ act on $X$ by $g\left(i, G_{i} x\right)=\left(i, G_{i} \times g^{-1}\right)$. Then $P(G, X, Y)$ is a proper inverse semigroup.

Conversely, if $S$ is a proper inverse semigroup then $S \approx P(G, X, Y)$, constructed as above, for some group $G$, set of subgroups $\left\{G_{i}: i \in I\right\}$ and sets of elements $E_{i}, B_{i j}$ such that (1), $\cdot ; ; ;$ (5) bold.

Proper ordered inverse semigroups were considered, and their structure was obtained, by Saitô [18]. Proper inverse semigroups have also been characterised by D. G. Green [2] in terms of the coordinatisation described in Proposition 1.1. Residuated inverse semigroups were considered by T. J. Blyth [1]; in this paper, he showed that a residuated inverse semigroup had an identity and that each $\sigma$-class had a maximum member. Semigroups with the latter property were called $F$-inverse semigroups by McFadden and $O^{\prime}$ Carroll [9] and a structure theorem for these semigroups, which are easily seen to be proper, is given there.

Since $F$-inverse, and, a fortiori, residuated inverse semigroups, are proper, they can be described in terms of the theory in this paper. 
Theorem 2.8. Let $\mathcal{X}$ be a semilattice and let $\mathcal{Y}$ be a principal ideal of $\mathcal{X}$ with greatest element $E$. Let $G$ be a group which acts on $\mathcal{X}$ by order automorphisms. Then $P(G, X, Y)$ is an $F$-inverse semigroup.

Conversely, if $S$ is an F-inverse semigroup, then $S \approx P(G, X, Y)$ for some $G, X, Y$ as above.

Proof. It follows from [8, Corollary 2.8] that $P(G, X, Y)$ is F-inverse if and only if $g E \wedge E$ exists for all $g \in G$. Hence $P(G, X, Y)$ is $F$-inverse if $X$ is a semilattice.

Conversely, if $S$ is $F$-inverse, it is proper and so, by Theorem 2.6, $S \approx$ $P(G, X, Y)$ for some $G, X, Y$. Further $S$ has an identity so that $\mathcal{Y}$ has a maximum element $E$ and we may suppose that $\mathcal{X}=G . Y$ and $Y \cap g . Y \neq q$ for each $g \in G$. Since $P(G, X, Y)$ is $F$-inverse, $[8$, Corollary 2.8] shows that $g E \wedge E$ exists for each $g \in G$. We shall use this to show that $X$ is a semilattice.

First, let $C \in \mathcal{X}$. Then there exists $g \in G$ such that $g . C \in \mathcal{Y}$. and so, since $y$ is a semilattice, g. $C \wedge(E \wedge$ g. $E)$ exists. But $g . C \leq E$ so that g. $C \wedge$ $\left(E \wedge g_{\cdot} E\right)=g_{\cdot} C \wedge_{g . E}$. Hence, since $G$ acts by order automorphisms, $C \wedge E$ exists.

Now let $A, B \in \mathcal{X}$. Then there exists $b \in G$ such that $b . B \in \mathcal{Y}$ and, by the immediately preceding paragraph, $b . A \wedge E$ exists. Thus $b . B \wedge(b . A \wedge E)$ exists and so, since $b_{.} B \leq E, b . B \wedge b . A$ exists. But $G$ acts by order automorphisms so the existence of $b . B \wedge b . A$ implies that of $B \wedge A$. Hence $\mathcal{X}$ is a semilattice.

Theorem 2.6 shows that every proper inverse semigroup can be described in terms of a group $G$ and a partially ordered set $\mathcal{X}$ which is nearly a semilattice; every principal ideal of $\mathcal{X}$ is a subsemilattice of $\mathcal{X}$. Theorem 2.8 shows, that, in general $\mathcal{X}$ cannot be a semilattice.

3. Idempotent separating congruences and arbitrary inverse semigroups, As a consequence of Theorem 2.6 and [6, Corollary 2.5], every inverse semigroup is an idempotent separating homomorphic image of some $P(G, X, Y)$. In this section we determine the idempotent separating congruences on $P(G, X, Y)$. Using these, we can give a structure theorem for arbitrary inverse semigroups in terms of groups and partially ordered sets.

$\Lambda$ set $\pi=\left\{N_{e}: e \in E\right\}$, of subgroups of an inverse semigroup $S$ with semilattice of idempocents $E$, is called a group kernel normal system if the following hold

(i) $e \in N_{e}$ for each $e \in E$;

(ii) $a^{-1} N_{e} a \subseteq N_{a-1}$ for each $a \in S$, $e \in E$;

(iii) $N_{e} N_{f} \subseteq N_{e f}$ for all $e, f \in E$. 
Let $r=\left\{N_{e}: e \in E\right\}$ be a group kernel normal system. Then Preston [13] has shown that the relation $\rho_{\pi}$ defined by $(a, b) \in \rho_{\pi} \Rightarrow a a^{-1}=b b^{-1}=e$ say and $a b^{-1} \in N_{e}$ is an idempotent separating congruence on $S$. Conversely, if $\rho$ is an idempotent separating congruence on $S$, then the set $\pi=\left\{N_{e}: e \in E\right\}$ of $\rho$-classes, which contain idempotents, is a group kernel normal system and $\rho=\rho_{\boldsymbol{r}^{\circ}}$

There is a largest group kernel normal system. This is the set $\mathcal{C}(E)=$ $\left\{C_{e}: e \in E\right\}$ where $C_{e}=\left\{a \in S: a a^{-1}=e, a f=f a\right.$ for all $\left.f^{2}=f \in S\right\}$; its union is the centraliser $C(E)$ of the idempotents of $S$. The corresponding largest idempotent separating congruence is

$$
\mu=\left\{(a, b) \in S \times S: a^{-1} e a=b^{-1} e b \text { for all } e^{2}=e \in S\right\}
$$

(Howie [3]); $\mu$ is the largest congruence contained in $\mathcal{H}$.

Suppose that $P(G, X, Y)$ is a $P$-semigroup and, for each $A \in Y$, let $C_{A}=$. ig $g \in G: g B=B$ for all $B \leq A\}$. Then $C_{A}$ is easily seen to be a subgroup of the stabilizer of $A$ under the action of $G$.

Proposition 3.1. Let $P(G, X, Y)$ be a P-semigroup and let $(A, g),(B, b) \epsilon$ P. Then $(A, g) \mu(B, b) \Leftrightarrow A=B$ and $g b^{-1} \in C_{A}$.

Proof. For $C \in Y,(A, g)^{-1}(C, 1)(A, g)=\left(g^{-1}(A \wedge C), 1\right)$. Hence, since $\mu \subseteq \mathcal{H}$

$(A, g) \mu(B, h) \Leftrightarrow A=B$ and $g^{-1}(A \wedge C)=b^{-1}(A \wedge C)$ for all $C \in y$

$$
\begin{aligned}
& \Leftrightarrow A=B \text { and } g^{-1} C=b^{-1} C \text { for all } C \leq A \\
& \Leftrightarrow A=B \text { and } g b^{-1} \in C_{A} .
\end{aligned}
$$

Group kernel normal systems can similarly be described in terms of $G$ and $Y$.

Proposition 3.2. Let $P(G, X, Y)$ be a P.semigrou $p$ and, for each $A \in \mathcal{Y}$, let $N_{A}$ be a subgroup of $C_{A}$ sucb that

(1) if $(C, g) \in P$ where $C \leq A$, then $g^{-1} N_{A} g \subseteq N_{g^{-1} C}$;

(2) $N_{A} N_{B} \subseteq N_{A \wedge B}$

Then $\pi=\left\{\{A\} \times N_{A}: A \in Y\right\}$ is a group kernel normal system and eacb sucb bas this form.

Proof. This is straightforward.

As a consequence of Theorem 2.6 and [6, Corollary 2.6], we now have the following structure theorem for arbitrary inverse semigroups.

Theorem 3.3. Let $\mathcal{X}$ be a partially ordered set witb $\mathcal{Y}$ an ideal and subsemilattice of $\mathcal{X}$ and let $G$ be a group wbicb acts on $X$ by order automorphisms. For eacb $A \in Y$, let $N_{A}$ be a subgroup of $C_{A}=\left\{g \in G:{ }_{g} C=C\right.$ for all $\left.C \leq A\right\}$ sucb 
that

(1) for each $g \in G, C \leq A$ such that $g^{-1} C \in Y, g^{-1} N_{A} g \subseteq N_{g^{-1} C}$;

(2) for $A, B \in Y, N_{A} N_{B} \subseteq N_{A \wedge B^{*}}$

Let $G_{A}=H_{A} / N_{A}$ where $H_{A}=\{g \in G: g A=A\}$ is the stablizer of $A$ under $G$. Then the set $I\left(G, \mathcal{X}, Y_{;} \gamma\right)$ of all pairs $(A, g) \in Y_{\times} G$ sucb that $g^{-1} A \in Y_{\text {is }}$ an inverse semigroup under the multiplication $(A, g)(B, b)=(A \wedge g B, g b)$ where we identify $(A, g)$ with $(B, b)$ if and only if $A=B$ and $g b^{-1} \in N_{A}$. It has semilattice of idempotents isomorphic to $Y$ and maximal subgroups isomorphic to $G_{A}, A \in$ Y.

Conversely, if $S$ is an inverse semigroup with semilattice of idempotents isomorpbic to $Y$ and maximal subgroups $G_{A}, A \in Y$ then $S \approx I(G, X, Y ; \mathcal{Y})$ for some $G, X$ and $\Re$, satisfying (1) and (2), as above with $G_{A} \approx H_{A} / N_{A}$ for each $A \in \mathcal{Y}$.

Proof. The first part is immediate from Proposition 3.2. Conversely, it follows from Theorem 2.6 and [6, Corollary 2.6], that there is a $P$-semigroup $P(G, X, Y)$ and an idempotent separating homomorphism $\psi$ of $P(G, X, Y)$ onto S. If $\pi$ is the group kernel normal system of this congruence then $S \approx$ $I(G, X, Y ; r)$. Further, it is easy to see that the maximal subgroup of $I(G, X, Y ; g)$ which contains $(A, 1)$ is isomorphic to $H_{A} / N_{A}$.

4. Fundamental inverse semigroups. An inverse semigroup $S$ is called fundamental if $\mu$ is the identity congruence on $S$. Munn [12] has shown that $S / \mu$ is fundamental for every inverse semigroup $S$. Thus we can use Proposition 3.1 to obtain a structure theorem for fundamental inverse semigroups analogous to Theorem 3.3.

Theorem 4.1. Let $\mathcal{X}$ be a partially ordered set with $\mathcal{Y}$ an ideal and subsemilattice of $\mathcal{X}$ and let $G$ be a group which acts on $\mathcal{X}$ by order automorphisms. Then the set $F(G, X, Y)$ of all pairs $(A, g) \in \mathcal{Y} \times G$, sucb that $g^{-1} A \in Y$, is an inverse semigroup under the multiplication $(A, g)(B, b)=(A \wedge g B, g b)$ where we identify $(A, g)$ and $(B, b)$, if and only if $A=B$ and $g C=b C$ for all $C \leq A$. Furtber $F(G, X, Y)$ is a fundamental inverse semigroup with semilattice of idem. potents isomorpbic to $\mathcal{Y}$.

Conversely, if $S$ is a fundamental inverse semigroup with semilattice of idempotents isomorpbic to $\mathcal{Y}$, then $S \approx F(G, X, Y)$ for some $G, X$.

Proof. The direct part is immediate from Proposition 3.1 and the fact that $S / \mu$ is fundamental for each inverse semigroup $S$.

Conversely, let $S$ be fundamental inverse semigroup. Then there is a $P$. semigroup $P(G, X, Y)$ and an idempotent separating homomorphism $\psi$ of 
$P(G, X, Y)$ onto $S$. Since $S$ is fundamental $\psi \circ \psi^{-1}$ is a maximal idempotent separating congruence on $P(G, Y, Y)$; thus $\psi \circ \psi^{-1}=\mu$ and, by Proposition 3.1, $S \approx F(G, X, Y)$. Finally, since $\psi$ is idempotent separating, $Y$ is isomorphic to the semilattice of idempotents of $S$.

As was the case in Theorem 3.3, the $P$-semigroup $P(G, X, Y)$ is not uniquely determined by the fundamental inverse semigroup $F(G, X, 9)$ to which it gives rise. Indeed, one can always assume that the group $G$ is a subgroup of the group of automorphisms of $X$. For each $g \in G$, let $\bar{g} \in$ Aut $X$ be defined by $x \vec{g}=g^{-1} \cdot x$ for each $x \in X$ and let $\bar{G}=\{\bar{g}: g \in G\}$ act on $X$ by $g \cdot x=x \bar{g}^{-1}$. Then the map $(A, g) \rightarrow(A, \bar{g})$ is easily seen to be an idempotent separating homomorphism of $P(G, X, Y)$ onto $P(\bar{G}, X, Y)$ and so $F(G, X, Y) \approx F(\bar{G}, X, Y)$.

Let $S$ be an inverse semigroup with semilattice of idempotents $E$ and, for each $a \in S$, define a partial one-to-one transformation $\mu_{a}$ of $E$ by $e \mu_{a}=a^{-1} e a$ for each $e \leq a a^{-1}$. Then Munn [12] showed that $\bar{\mu}: a \mapsto \mu_{a}$ is a homomorphism of $S$ onto a full subsemigroup of the inverse semigroup $T_{E}$ of isomorphisms between principal ideals of $E$. Further $\bar{\mu} \circ \bar{\mu}^{-1}$ is the maximum idempotent separating congruence on $S$.

The representation $\bar{\mu}$ can be nicely exhibited for $P$-semigroups. If $P=$ $P(G, X, Y)$ and $a=(A, g)$ then $\mu_{a}$ is the partial one-to-one transformation of $y$ defined by $B \mu_{a}=g^{-1} B$ for all $B \leq A$.

The theory in this paper gives rise to an interesting result on semilattices which it seems appropriate to include here.

Theorem 4.2. Let $E$ be a semilattice. Then $E$ can be embedded as an ideal in a partially ordered set $X$ with the following property: Every isomorphism be. tween principal ideals of $X$ can be extended to an automorpbism of $X$.

Proof. By Theorem 2.6 and [6, Corollary 2.5], there is a $P$-semigroup $P=$ $P(G, X, E)$, with $X=G . E$, such that the mapping $\bar{\mu}: P \rightarrow T_{E}$, defined by $(A, g) \bar{\mu}=\bar{g}$ restricted to $\{B \in \mathcal{Y}: B \leq A\}$, is an idempotent separating homomorphism of $P$ onto $T_{E}$, where $B \bar{g}=g^{-1} \cdot B$ for each $B \in X$.

Suppose $\beta$ is an isomorphism of $\{z \in \mathfrak{X}: z \leq x\}$ onto $\{z \in \mathfrak{X}: z \leq y\}$. Since $X=G . E$, there exist $g, b \in G$ such that $e=g \cdot x \in E, f=b \cdot y \in E$. The map $a$ defined by $u a=u \bar{g}^{-1} \beta \bar{b}$ for each $u \leq e$ is then an isomorphism of $\{v \in E: v \leq e\}$ onto $\{v \in E: v \leq f\}$ and so belongs to $T_{E}$. Hence $\alpha=(e, k) \bar{\mu}$ for some $k \in G$. But this means $u a=u \bar{k}$ for each $u \leq e$ and so $v \beta=v \bar{g} a \bar{b}^{-1}=v \bar{g} \bar{k} \bar{b}^{-1}$ for each $v=u \bar{g}^{-1} \leq x$. Hence $\bar{g} \bar{k} \bar{b}^{-1}$ is an automorphism of $X$ which extends $\beta$.

5. An application. In this section, we give an example to show that most of the structure theorems, which have been obtained for inverse semigroups, can be deduced from Theorem 3.3. The example chosen is that of an $\omega$-bisimple semi- 
group [14]. The structure theorems for arbitrary bisimple inverse semigroups [5], for $\omega$-simple inverse semigroups [4], [11] and l-simple inverse semigroups [19] . can be obtained in a similar way.

An inverse semigroup $S$ is $\omega$-bisimple if it is bisimple and its idempotents form a chain isomorphic to the nonpositive integers $\mathrm{Z}^{-}$under the usual ordering. Thus, by Theorem 3.3 , if $S$ is an $\omega$-bisimple semigroup then $S \approx I\left(G, \mathcal{X}, \mathrm{Z}^{-} ; \boldsymbol{r}\right)$ for some partially ordered set $\mathcal{X}$ containing $Z^{-}$as an ideal, some group $G$ acting transitively on $\mathcal{X}$ by order automorphisms and some family $\pi$ of subgroups of $G$ which satisfy (1) and (2) of Theorem 3.3; $G$ acts transitively because $S$ is bisimple.

Because $G$ acts transitively, there exists $g \in G$ such that $g^{-1} .0=-1$; then $a=(0, g) \in P=P\left(G, X, Z^{-}\right)$. The following lemma contains some elementary properties of $g$ which will be useful in the sequel.

Lemma 5.1. Let $m \geq 0$. (i) If $-n \leq 0$ then $g^{-m} \cdot-n=-(m+n)$. (ii) If $-n \leq-m$ then $g^{m} .-n=-n+m$. (iii) If $-n \leq 0$ and $h .0=0$ then $h .-n=-n$.

Proof. (i) Since $g^{-1}$ acts by order automorphisms it induces an order isomorphism of $Z^{-}$onto $\left\{x \in Z^{-}: x \leq-1\right\}$. Hence $g^{-1} \cdot-x=-(x+1)$ for any $x \in \mathrm{Z}^{-}$and so, by induction, $g^{-m}$. $-n=-(m+n)$.

(ii) If $-n=-(m+r)$ then, by (i), $-n=-(m+r)=g^{-m} \cdot-r$ and so $\boldsymbol{g}^{m} \cdot-n=-r=-n+m$.

(iii) If $b .0=0$ then $b$ induces an order isomorphism of $\mathrm{Z}^{-}$onto $\mathrm{Z}^{-}$. Hence, since the identity is the only such, $b_{0}-n=-n$ for all $-n \leq 0$.

Corollary 5.2. For any $n \in \mathrm{Z}^{+}$,

$$
a^{n}=\left(0, g^{n}\right), \quad a^{-n}=\left(-n, g^{-n}\right), \quad a^{-n} a^{n}=(-n, 1)
$$

and, for $b \equiv(0, b)$ where $b \in H=\{b \in G: b .0=0\}, a^{-m} b a^{n}=\left(-m, g^{-m} b g^{n}\right)$.

It follows from Corollary 5.2 that the elements $a^{n}, n \in \mathrm{Z}^{+}$are representatives of the $\mathcal{H}$-classes of $P$ contained in the R-class of $(0,1)$. Hence, as at the beginning of $\$ 2$, each element of $P$ can be uniquely expressed in the form $a^{-m} b a^{n}$. The idea is use this coordinatisation to obtain the usual structure theorem for $S$ [14].

Let $U$ be the group of units of $S$. Then, since $S \approx I\left(G, X, Z^{-} ; r\right)$, there is a homomorphism $\phi$ of $H$ onto $U$ with kernel $N_{0}$.

Lemma 5.3. $N_{-m}=g^{-m} N_{0} g^{m}$ for any $m \leq 0$.

Proof. By Proposition 3.2(1), 


$$
g^{-m} N_{0} g^{m} \subseteq M_{8^{-m} .0}=N_{-m}
$$

since $\left(0, g^{m}\right) \in P$. Similarly, since $\left(-m, g^{-m}\right) \in P$,

$$
g^{m} N_{-m} g^{-m} \subseteq N_{8^{m} \cdot-m}=N_{0}
$$

Hence $N_{-m}=g^{-m} N_{0} g^{m}$.

Because each element of $P$ is uniquely of the form $a^{-m} b a^{n}$, Corollary 5.2 shows that we can define a map $\psi$ of $P$ onto $Z^{+} \times U \times Z^{+}$by $\left(-m, g^{-m} b g^{n}\right) \psi=$ $(m, b \phi, n)$. Then

$$
\begin{aligned}
&\left(-m, g^{-m} b g^{n}\right) \psi=\left(-p, g^{-p} k g^{q}\right) \psi \Leftrightarrow m=p, n=q, b \phi=k \phi \\
& \Longrightarrow m=p, n=q, b k^{-1} \in N_{0^{\circ}}
\end{aligned}
$$

Thus, by Lemma 5.3,

$\left(-m, g^{-m} b g^{n}\right) \psi=\left(-p, g^{-p} k g^{q}\right) \psi \Leftrightarrow m=p, n=q$ and $g^{-m} b g^{n}\left(g^{-p} k g^{q}\right)^{-1} \in N_{-m}$;

that is, if and only if they are equal in $(G, X, Y ; \pi)$.

In order to obtain a structure theorem for $S \approx \mathbb{N}(G, \mathcal{X}, \mathcal{Y} ; r)$ we need thus only define a multiplication on $\mathrm{Z}^{+} \times U \times \mathrm{Z}^{+}$in such a way that $\psi$ becomes a homomorphism.

Let $\beta$ be defined by $b \beta=g h g^{-1}$ for each $b \in H$. Then it is easyy to see that $\beta$ is a one-to-one homomorphism of $H$ into itself. Further, since $\left(-1, g^{-1}\right) \epsilon$ $P, N_{0} \beta=g N_{0} g^{-1} \subseteq g N_{-1} g^{-1}=N_{0} ; N_{0} \subseteq N_{-1}$ by Proposition 3.2. It follows that the diagram

$$
\underset{U}{H} \stackrel{\beta}{\longrightarrow} \underset{U}{\downarrow_{U}^{H}}
$$

can be uniquely completed to a commutative diagram by an endomorphism $a$ of $U$. Now

$$
\begin{aligned}
& \left(-m, g^{-m} b g^{n}\right)\left(-p, g^{-p} k g^{q}\right)=\left(-m \wedge g^{-m} b g^{n} .-p, x\right) \\
& =\left(g^{-m} b g^{n}\left(g^{-n} b^{-1} g^{m} \cdot-m \wedge-p\right), x\right)=\left(g^{-m} b g^{n}(-n \wedge-p), x\right)
\end{aligned}
$$

by Lemma 5.1,

$$
=((-n \wedge-p)+n-m, x)=(-(p \vee n-: n+m), x)
$$

where $x=g^{-m} b g^{n} g^{-p} k g^{q} ; p \vee n$ denotes the maximum of $p$ and $n$. But

$$
\begin{aligned}
x & =g^{-m} b g^{n} g^{-p} k g^{q} \\
& =g^{-(p \vee n-n+m)} g^{p \vee n-n} b g^{-(p \vee n-n)} g^{p \vee n-p} k g^{-(p \vee n-p)} g^{p \vee n-p+q} \\
& =g^{-(p \vee n-n+m)} b \beta^{p \vee n-n} k \beta^{n \bigvee p-p} g^{n \bigvee p-p+q} .
\end{aligned}
$$


Hence

$$
\begin{aligned}
{\left[\left(-m, g^{-m} b g^{n}\right)\right.} & \left.\left(-p, g^{-p} k g^{q}\right)\right] \psi \\
= & \left(p \vee n-n+m,\left[b \beta^{p \vee n-n} k \beta^{n \vee p-p}\right] \phi, n \vee p-p+q\right) \\
= & \left.(p \vee n-n+m) b a^{p \vee_{n-n}} k \phi a^{n \vee p-p}, n \vee p-p+q\right)
\end{aligned}
$$

since $\beta \phi=\phi a$. It follows that $\psi$ becomes an isomorphism if we define multiplication on $\mathbf{Z}^{+} \times U \times \mathbf{Z}^{+}$by

$$
(m, u, n)(p, v, q)=\left(p \vee n-n+m, u a^{p \vee n-n} v a^{n \vee p-p}, n \vee p-p+q\right)
$$

This is precisely the multiplication in Reilly's structure theorem for $S$; the semigroup $\mathrm{Z}^{+} \times U \times \mathrm{Z}^{+}$with this multiplication is denoted by $B(U, a)$.

Theorem 5.4 [14]. Every $\omega$-bisimple semigroup is isomorpbic to some $B(U, \alpha)$.

The converse of Theorem 5.4 is also true [14].

The other structure theorems for inverse semigroups can be obtained in a similar way by coordinatising $P(G, X, Y)$ as in $\$ 2$ in the form $r_{i u}^{-1} b_{i} r_{i v}$ and considering the map $\psi: P \rightarrow E \times \bigcup\left\{U_{i}: i \in I\right\} \times E$ where $\left.S \approx \Lambda G, \mathcal{X}, Y_{;}, \mathcal{I}\right)$ and $U_{i} \approx H_{i} / N_{f_{i}}$ is the maximal subgroup of $S$ containing the image of $\left(f_{i}, 1\right)$.

6. Homomorphisms of $P$-semigroups. Let $X_{i}$ be a partially ordered set with $Y_{i}$ an ideal and subsemilattice of $\mathscr{X}_{i}$ and let $G_{i}$ be a group which acts on $\mathcal{X}_{i}$ by order automorphisms, $i=1,2$, such that P.1 and P. 2 of $\$ 1$ hold. By a morphism of the action of $G_{1}$ on $X_{1}$ to the action of $G_{2}$ on $X_{2}$ we mean a pair $(\theta, \phi)$ where

(1) $\theta$ is an isotone mapping of $x_{1}$ into $x_{2}$ such that $y_{1} \theta \subseteq y_{2}$ and $(A \wedge B) \theta=A \theta \wedge B \theta$ for all $A, B \in y_{1}$;

(2) $\phi$ is a homomorphism of $G_{1}$ into $G_{2}$;

(3) the diagram

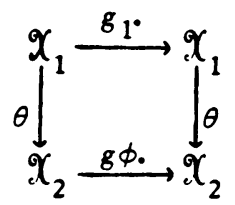

commutes for each $g \in G$; that is $(g . A) \theta=g \phi . A \theta$ for all $g \in G_{1}, A \in X_{1}$.

If $(\theta, \phi)$ is such a morphism, it is easy to see that the mapping $\psi: P_{1} \rightarrow P_{2}$ defined by $(A, g) \psi=(A \theta, g \phi)$ is a homomorphism. On the other hand, the proof of Theorem 1.3 shows that, if $\psi: P_{1} \rightarrow P_{2}$ is a homomorphism, there is a (unique) morphism $(\theta, \phi)$ of the action of $G_{1}$ on $X_{1}$ to the action of $G_{2}$ on $X_{2}$ such that $(A, g) \psi=(A \theta, g \phi)$ for all $A \in X_{1}, g \in G_{1}$. Hence we have 
Theorem 6.1. Let $\mathfrak{X}_{i}$ be a partially ordered set with $\mathfrak{Y}_{i}$ an ideal and subsemilattice of $X_{i}$ and let $G_{i}$ be a group which acts on $X_{i}$ by order automorphisms such that P.1 and P.2 of $\$ 1$ bold, $i=1,2$. Then there is a one-to-one correspondence between the set of bomomorphisms from $P\left(G_{1}, X_{1}, \mathscr{Y}_{1}\right)$ to $P\left(G_{2}, X_{2}, Y_{2}\right)$ and the set of morphisms of the action of $G_{1}$ on $X_{1}$ to the action of $G_{2}$ on $X_{2}$.

By Theorem 2.6, every proper inverse semigroup is a $P$-semigroup. Thus Theorem 6.1 describes the homomorphisms between proper inverse semigroups. We can also use Theorem 6.1 to characterise those congruences on $P$-semigroups whose quotients are proper inverse semigroups.

Proposition 6.2. Let $\mathcal{X}$ be a partially ordered set with $\mathcal{Y}$ an ideal and sub. semilattice of $X$. Let $G$ be a group which acts on $\mathcal{X}$ by order automorphisms and suppose that

(P.1) G. $Y=\mathscr{X}$,

(P.2) $g . \mathcal{Y} \cap \mathcal{Y} \neq \square$ for each $g \in G$.

Suppose further that $\rho, \pi$ are equivalences on $X$ and $G$ such that $\pi$ is a con. gruence and

$$
\begin{aligned}
(A, B) \in \rho,(g, b) \in \pi & \Rightarrow(g A, b B) \in \rho, \\
A, B, C \in Y,(A, B) \in \rho & \Rightarrow(A \wedge C, B \wedge C) \in \rho .
\end{aligned}
$$

Then $\tau$ defined by $(A, g) \tau(B, b) \Leftrightarrow(A, B) \in \rho,(g, b) \in \pi$ is a congruence on $P=P(G, X, Y)$ such that $P / \tau$ is proper. Conversely, every sucb bas this form.

Proof. Given $\rho, \pi$, it is straightforward to show that $r$ is a congruence and that $P / \tau$ is proper.

Conversely, if $\tau$ is such a congruence then, by Theorem 2.6 we may suppose $P / \tau=P(H, \mathcal{U}, \mathcal{O})$. Hence, by Theorem 6.1 , there is a morphism $(\theta, \phi)$ of the action of $G$ on $\bar{X}$ to the action of $H$ on $\mathcal{U}$ such that $(A, g) \tau^{h}=(A \theta, g \phi)$. Let $\rho=\theta \circ \theta^{-1}, \pi=\phi \circ \phi^{-1}$. Then clearly

$$
(A, g) \tau(B, b) \Leftrightarrow(A, B) \in \rho,(g, b) \in \pi .
$$

Further $\pi$ is a congruence and, by (1) of the definition of morphism $A, B, C \in \mathcal{Y}$, $(A, B) \in \rho$ implies $(A \wedge C, B \wedge C) \in \rho$; by (3) of the definition of morphism, $(A, B) \in \rho,(g, b) \in \pi \Rightarrow(g A, b B) \in \rho$.

An equivalence relation $\eta$ on the set $E$ of idempotents of an inverse semigroup $S$ is called a normal partition of $E$ if $\eta$ is the restriction to $E \times E$ of a congruence on $S$. Reilly and Scheiblich [16] have shown that an equivalence $\eta$ on $E$ is a normal partition if and only if 


$$
\begin{aligned}
& (e, f) \in \eta, g \in E \Rightarrow(e g, f g) \in \eta, \\
& (e, f) \in \eta, a \in S \Rightarrow\left(a^{-1} e a, a^{-1} f a\right) \in \eta
\end{aligned}
$$

Corresponding to a normal partition $\eta$ on $E$ there is a smallest congruence $\eta^{*}$ on $S$ whose restriction to $E \times E$ is $\eta$; this is defined by

$$
(a, b) \in \eta^{*} \Leftrightarrow a a^{-1} \eta b b^{-1} \eta e \text { for some } e \in E \text { such that } e a=e b
$$

Lemma 6.3. Let $S$ be a proper inverse semigroup and let $\eta$ be a normal partition on the set $E$ of idempotents of $S$. Let $T=S / \eta^{*}$ and for eacb $s \in S$, let $\bar{s}$ denote the image of $s$ in $T$. Then

$$
(\bar{s}, \bar{t}) \in \sigma \Leftrightarrow(s, t) \in \sigma .
$$

In particular $T / \sigma \approx S / \sigma$ and $T$ is proper.

Proof. If $(s, t) \in \sigma$ then clearly $(\bar{s} ; \bar{t}) \in \sigma$. On the other hand, $(s, t) \in \sigma$ implies (es, et) $\in \eta^{*}$ for some $e \in E$ and, by the definition of $\eta^{*}$ this means $e s(e s)^{-1} \eta e t(e t)^{-1} \eta f$ for some $f \in E$ such that fes $=f e t$. But $f e$ is idempotent so fes $=$ fet implies $(s, t) \in \sigma$.

It is immediate from $(*)$ that $T / \sigma \approx S / \sigma$. Suppose $\left(\bar{s}^{2}, \bar{s}\right) \in \sigma$ then $\left(s^{2}, s\right) \in \sigma$. and so since $S$ is proper $s^{2}=s$. Hence $\left(\bar{s}^{2}, \bar{s}\right) \in \sigma$ implies $\bar{s}^{2}=\bar{s}$ and so $T$ is proper.

It follows from Lemma 6.3 that if $\eta$ is a normal partition on the idempotents of $P=P(G, X, Y)$ then $P / \eta^{*}$ is proper and so, by Theorem $2.6, \eta^{*}$ is of the form described in Proposition 6.2. Thus we can obtain

Proposition 6.4. Let $\mathcal{X}$ be a partially ordered set witb $\mathcal{Y}$ an ideal and sub. semilattice of $\mathcal{X}$ and let $G$ be a group which acts on $\mathcal{X}$ by order automorphisms. Suppose that $\mathcal{X}=G . \mathcal{Y}$ and $g . \mathcal{Y} \cap Y \neq \square$ for any $g \in G$. Let $\rho$ be an equivalence relation on $\mathcal{X}$ such that

$$
(A, B) \in \rho \Rightarrow(g A, g B) \in \rho \text { for all } g \in G
$$

$$
\text { if } A, B, C \in Y \text { and }(A, B) \in \rho \text { then }(A \wedge C, B \wedge C) \in \rho \text {. }
$$

Then $\eta$ defined by

$$
(A, 1) \eta(B, 1) \leftrightarrow A, B \in Y \text { and }(A, B) \in \rho
$$

is a normal partition on the idempotents of $P(G, X, Y)$; further

$$
(A, g) \eta^{*}(B, h) \leftrightarrow(A, B) \in \rho \text { and } g=h \text {. }
$$

Conversely, if $\eta$ is a normal partition on the idempotents of $P$ then $\eta$ and $\eta^{*}$ bave the form above for some equivalence $\rho$ on $X$. 
Proof. Given $\rho$, it is a straightforward matter to verify that $\eta$ is a normal partition and that $\eta^{*}$ has the form described.

Conversely, if $\eta$ is a normal partition, it follows from Lemma 6.3 that $P / \eta^{*} \approx P(G, \mathcal{U}, \mathcal{O})$ for some $\mathcal{U}$ and $\mathcal{O}$ and so, by Proposition 6.2 , there is an equivalence $\rho$ on $X$, which satisfies the conditions in the statement of the proposition, such that

$$
(A, g) \eta^{*}(B, h) \Leftrightarrow(A, B) \in \rho, \quad g=b ;
$$

$\pi$ is the identity congruence on $G$, by Lemma 6.3 . Then

$$
(A, 1) \eta(B, 1) \Leftrightarrow(A, B) \in \rho .
$$

If $\rho$ is an equivalence on $X$, as in Proposition 6.4 , then $P / \eta^{*}$ is easy to describe. It consists of all pairs $(A, g) \in \mathscr{Y}_{\times} \in$ under the multiplication $(A, g)(B, b)=(A \wedge g B, g b)$ where $(A, g)$ is identified with $(B, b)$ if and only if $(A, B) \in \rho, g=b$. Thus $P / \eta^{*}$ is the $P$-semigroup $P(G, \mathcal{U}, Y / \rho)$ for some partially ordered set containing $Y / \rho$ as an ideal. The conditions in Proposition 3.3 for idempotent separating congruences on $P(G, X, Y)$ make use only of the part of $\mathcal{X}$ contained in G.Y. Hence the idempotent separating congruences on $P / \eta$ * can be described without explicitly knowing $\mathcal{U}$. Thus one can construct all congruences on P-semigroups. By Theorem 3.3, one can use similar arguments to find the congruences on arbitrary inverse semigroups.

\section{REFERENCES}

1. T. S. Blyth, Residuated inverse semigroups, J. London Math. Soc. (2) 1 (1969), 243-248. MR 39 \# 5439.

2. D. G. Green, Extensions of a semilattice by an inverse semigroup, Bull. Austral. Math. Soc. 9 (1973), 21-32.

3. J. M. Howie, The maximum idempotent-separating congruence on an inverse semigroup, Proc. Edinburgh Math. Soc. (2) 14 (1964/65), 71-79. MR 29 \# 1275.

4. B. P. Kočin, The structure of inverse ideal-simple w-semigroups, Vestnik Leningrad Univ. 23 (1968), no. 7, 41-50. (Russian) MR 37 \# 2881. appear).

5. D. B. McAlister, O-bisimple inverse semigroups, Proc. London Math. Soc. (to

6. —, Groups, semilattices and inverse semigroups, Trans. Amer. Math. Soc. (to appear).

7. - Groups, semilattices and inverse semigroups, Proc. Sympos. on Inverse Semigroups, Northern Illinois University, DeKalb, Ill., 1973.

8. D. B. McAlister and R. McFadden, Zig-zag representations and inverse semigroups, J. Algebra (to appear).

9. R. McFadden and L. O'Carroll, F-inverse semigroups, Proc. London Math. Soc. (3) 22 (1971), 652-666. MR 45 \# 2059.

10. W. D. Munn, A class of irreducible matrix representations of an arbitrary inverse semigroup, Proc. Glasgow Math. Assoc. 5 (1961), 41-48. MR 27 \# 3723. 
11. W. D. Munn, Regular wosemigroups, Glasgow Math. J. 9 (1968), 46-66. MR 37 \#3316.

12. - Fundamental inverse semigroups, Quart. J. Math. Oxford Ser. (2) 21 (1970), 157-170. MR $41 \# 7010$.

13. G. B. Preston, Inverse semigroups, J. London Math. Soc. 29 (1954), 396-403. MR 16, 215.

14. N. R. Reilly, Bisimple w-semigroups, Proc. Glasgow Math. Assoc. 7 (1966), 160-167. MR $32 \# 7665$.

15. - Congruences on a bisimple inverse semigroup in terms of RP-systems, Proc. London Math. Soc. (3) 23 (1971), 99-127. MR 46 \#5501.

16. N. R. Reilly and H. E. Scheiblich, Congruences on regular semigroups, Pacific J. Math. 23 (1967), 349-360. MR 36 \# 2725.

17. H. E. Scheiblich, Free inverse semigroups, Proc. Amer. Math. Soc. 38 (1973), $1-7$.

18. T. Saitô, Proper ordered inverse semigroups, Pacific J. Math. 15 (1965), 649666. MR 33 \# 204.

19. R. J. Wame, I-re gular semigroups, Math. Japon. 15 (1970), 91-100. MR 44 \#5398.

DEPARTMENT OF MATHEMATICAL SCIENCES, NORTHERN ILLINOIS UNIVERSITY, DEKAL B, ILLINOIS 60115 